\title{
Características epidemiológicas, clínicas y microbiológicas de las infecciones nosocomiales urinarias en las lesiones medulares espinales
}

\author{
Hernández González E*, Zamora Pérez F*, Martínez Arroyo M**, Valdez Fernández M*, \\ Alberti Amador E*. \\ ${ }^{*}$ Centro Internacional de Restauración Neurológica, **Departamento de Microbiología. Centro de Investigaciones \\ Médico-Quirúrgicas. Habana, Cuba.
}

Actas Urol Esp. 2007;31(7):764-770

\section{RESUMEN}

CARACTERÍSTICAS EPIDEMIOLÓGICAS, CLÍNICAS Y MICROBIOLÓGICAS DE LAS INFECCIONES NOSOCOMIALES URINARIAS EN LAS LESIONES MEDULARES ESPINALES

Las infecciones urinarias constituyen una de las más importantes causas de infecciones intrahospitalarias. En la clínica de atención a pacientes lesionados medulares espinales (LME) observamos que las mismas alcanzan una alta incidencia como consecuencia de múltiples factores de riesgo asociados con la vejiga neurogénica como son: reflujo vésico-ureteral, litiasis renal o vesical, divertículos y pseudodivertículos, estenosis uretral y el uso de catéteres vesicales permanentes o intermitentes.

Objetivos: Describimos las formas clínicas de presentación de las Infecciones del Tracto Urinario (ITU) en pacientes con lesiones medulares espinales con vejiga neurogénica así como el comportamiento microbiológico de las mismas.

Pacientes y Método: Realizamos un estudio descriptivo, de tipo retrospectivo a 28 pacientes hospitalizados por afección medular espinal y que se encontraban en evaluación para realizar tratamiento neuro- restaurativo. A los mismos se les realizó evaluación clínica y estudios imagenológicos y microbiológicos del tracto urinario y urocultivo, exudado vaginal y uretra para determinar los factores de riesgo, formas de presentación de la infección así como complicaciones asociadas además del comportamiento microbiológico.

Resultados: La forma más frecuente de presentación de las ITU fueron: bacteriuria sintomática recurrente, bacteriuria asintomática, uretritis bacteriana, vaginosis bacteriana y pielonefritis aguda. Los gérmenes aislados fueron: $E$. coli en el 60\% de los aislamientos, seguido por P. mirabilis en el 14\%, K. pneumoniae 10\% y Staphylococcus sp en el $4 \%$ así como otras enterobacterias. La sensibilidad a los aminoglucósidos se mantiene alta, aunque se observa una creciente resistencia a las sulfas ( $>70 \%)$ y a las fluoroquinolonas (>45\%) además de incrementarse la circulación de uropatógenos multirresistentes.

Conclusiones: Las particularidades clínicas de las UTI en pacientes con vejiga neurogénica por lesión de la médula espinal necesita adecuadas estrategias para el manejo clínico, microbiológico y epidemiológico de las mismas.

Palabras claves: Infección urinaria. Paraplejia. Lesión medular espinal. E.coli. Vejiga neurogénica.

\section{ABSTRACT \\ EPIDEMIOLOGIC, CLINICAL AND MICROBIOLOGICAL CHARACTERISTICS OF NOSOCOMIAL URINARY INFECTION IN THE SPINAL CORD LESIONED PATIENT}

Urinary infections constitute one of the main causes of intrahospitalary infections. At the Clinic for the attention of spinal cord injured (SCI) patients, we observed that these can be the causes of high incidence rates as a consequence of multiple risk factors associated with the neurogenic bladder as: vesical urethral reflux, vesicle lithiasis, diverticula and pseudodiverticula, urethral stenosis and permanent or intermittent catheterization.

Objectives: To describe forms of presentation of urinary tract infections (UTI) in spinal cord lesioned patients with neurogenic bladder as well as their microbiological behavior.

Patients and Method: We performed a descriptive, retrospective-type study on 28 patients in order to schedule a neurorestorative treatment for the affectation of the SCI for six months. They all received clinical, imaging test and bacteriologic assessment, that is, urocultures, uretheral and vaginal exudates to determine risk factors, forms of presentation of the infection, as well as associated complications and microbiological behavior.

Results: The most frequent forms of presentation of infections are: recurrent symptomatic bacteriuria, asymptomatic bacteriuria, bacterial urethritis, bacterial vaginosis and acute pyelonephrites. Most acute germs are: $E$ coli (for a $60 \%$ of isolation), followed by $P$. mirabilis (14\%), K pneumoniae (10\%), Staphylococcus $s p$. (4\%), and other enterobacteria. Sensitiveness to aminoglycosides was kept high, where we observed a growing resistance to sulphas $(>70 \%)$ and fluoroquinolones $(>45 \%)$ as well as the frequent circulation of multirresistant microorganisms.

Conclusions: Clinical peculiarities of urinary infections in the patient with neurogenic bladder, allow to perform more adequate strategies for treatment as to the clinical, microbiological and epidemiologic criteria.

Keywords: Urinary tract infections. Spinal cord injures. E. coli. Neurogenic bladder. 
$\mathrm{L}^{2}$ a infección urinaria tiene todavía una gran incidencia en el ambiente hospitalario, incluso con las tendencias actuales hacia ciertas reducciones y hacia un aumento de la incidencia de infecciones respiratorias hospitalarias, tal y como ha sido publicado en algunos estudios epidemiológicos españoles ${ }^{1}$. En nuestro ambiente la tendencia se ha comportado de forma variable, por ejemplo las infecciones urinarias nosocomiales en los últimos cinco años: en el año 2001 fueron el 65\% (infecciones respiratorias el 31\%), en 2002 el 57\% (infecciones respiratorias el 28,9\%), en 2003 un 46,5\% (infecciones respiratorias el $37,9 \%$ ), en 2004 el 37,8\% (infecciones respiratorias 39,3\%), mientras que en 2005 constituyeron el $43 \%$ (respiratorias 35,4\%). Estos datos se deben fundamentalmente a un estricto programa de vigilancia, prevención y control de infecciones, sobre todo en la clínica de enfermedades de la médula espinal -en nuestro hospital la unidad con la incidencia más alta- donde los pacientes siguen un programa neuroregenerativo con estancias prolongadas, con una tasa de infecciones nosocomiales comunicada de 15-20 episodios por cada 100 altas hospitalarias, y una incidencia de 4-5 episodios por 1.000 pacientes-días. Esto es significativo, porque en la era de la resistencia a antibióticos estos aspectos tienen un impacto mayor, sobre todo, porque se trata de pacientes con comorbilidad -como anomalias morfológicas y funcionales del aparato urinarioasociada a factores de riesgo por su vejiga neurógena, tales como cateterismos, reflujo vesicoureteral, litiasis y divertículos entre los más frecuentes; son clasificadas como infecciones complicadas, donde los uropatógenos pueden acceder a la vejiga urinaria, proliferar y producir una infección, bien sea por la turbulencia del flujo urinario durante el vaciado anormal consecuencia de la disfunción funcional o anatómica, o el resultado de una instrumentación o procedimiento invasivo, de uso frecuente en estos pacientes. Es una cuestión que favorece un acceso bacteriano más fácil al tracto urinario, su proliferación y por lo tanto la infección, tanto si se produce por uropatógenos que se adhieren a la célula epitelial del tracto urinario, como si es por medio de organelas con receptores específicos en el urotelio, tales como los pilli o las fimbrias del E. Coli. Esto puede también comprometer la eliminación de bacterias por los mecanismos normales de defensa, mecanismo frecuente de infección del tracto urinario en pacientes con vejiga neurógena. En niños portadores de E.coli con fimbrias en intestino, el riesgo de colonización del área periuretral por estos patógenos también está aumentado ${ }^{2-3}$.

\section{Tendencias actuales del comportamiento de las infecciones urinarias en el paciente lesionado médular.}

En el pasado, se comunicó una alta mortalidad en pacientes lesionados medulares durante la Segunda Guerra Mundial causada por pielonefritis e insuficiencia renal ${ }^{4-5}$, que alcanzaba hasta el $80 \%$. Sin embargo, la alta incidencia de lesión medular espinal a nivel mundial, que varía entre 9-53 casos por millón de habitantes hace que cada vez mucha más gente viva con las secuelas de una lesión medular. A pesar de la sustancial reducción de la mortalidad, e incluso de la morbilidad causada por las infecciones del tracto urinario $^{6}$, las secuelas de la disfunción establecida del esfinter y el factor de riesgo con ella asociado, éstas son todavía una razón importante de atención por parte de neurólogos, urólogos, internistas y psiquiatras.

El criterio tradicional de $\geq 10^{5} \mathrm{UFC} / \mathrm{mL}$ tiene baja sensibilidad, inaceptable para el diagnóstico clínico en pacientes lesionados medulares espinales.

La conferencia de consenso NIDRR recomendó el siguiente criterio de recuento de colonias de uropatógenos para el diagnóstico de bacteriuria significativa en lesionados medulares: $\geq 10^{2}$ $\mathrm{UFC} / \mathrm{mL}$ para muestras de catéteres de personas en programa de cateterismo intermitente; $\geq 10^{2}$ $\mathrm{UFC} / \mathrm{mL}$ para muestras de orina por micción limpia en varones que no utilizan catéter, sino, sistemas colectores de tipo condón; y cualquier concentración detectable de uropatógenos de aspirados suprapúbicos u obtenidos por sondas permanentes. Estos criterios cuantitativos del cultivo de orina tienen una sensibilidad y especificidad óptimas, y enfatizan la necesidad de considerar el tipo de drenaje vesical cuando se evalúa la bacteriuria ${ }^{7-9}$.

La incidencia de infección urinaria en pacientes con lesiones de la médula espinal varía de 
acuerdo con el método utilizado de vaciado o entrenamiento vesical, así como de los factores de riesgo asociados desde el punto de vista morfológico y funcional. Estos datos también incluyen las características socioeconómicas del paciente y la independencia funcional, razón por la que se comunican tasas de infección del $20 \%$, con variaciones en relación con el sistema de vaciado vesical; así la incidencia en algunas series es de 0,68 episodios por 100 pacientes-día, mientras que aquellos que utilizan sistemas de cateterismo limpio comunican 2,72 episodios por 100 pacientes-día. El sistema de vaciado mediante colectores o condones en hombres tiene una incidencia de 0,41 episodios por 100 pacientesdía, mientras que con los métodos de estimulación o Credè y maniobras de Valsalva en mujeres se comunica una incidencia de 0,36 episodios por 100 pacientes-día ${ }^{10-12}$.

$\mathrm{El}$ manejo adquiere ciertas características en esta población hospitalaria -como sus formas clínicas de presentación- con un incremento gradual de las resistencias microbianas y el subsiguiente fallo del tratamiento y recurrencia, lo cual contribuye a la frecuente circulación de cepas multirresistentes, así como al aumento de los costos económicos y humanos.

Por esa razón, en general los regímenes de tratamiento empírico y profiláctico no están recomendados en estos pacientes con infecciones complicadas. Es necesario conocer el comportamiento clínico, microbiológico y epidemiológico de la infección urinaria en el paciente lesionado médular y el uso de una premisa necesaria para el tratamiento y seguimiento adecuado que permita diseñar estrategias institucionales para encarar el desafio que la aparición de resistencias de los uropatógenos presenta en estas especialidades médicas para reducir las complicaciones crónicas asociadas, tales como el deterioro anatómico y funcional del tracto urinario, la cistitis crónica intersticial, el cáncer de vejiga y el deterioro de la función renal $^{3-14}$.

\section{OBJETIVOS}

- Definir las formas clínicas de presentación de las infecciones urinarias en pacientes lesionados medulares espinales.
- Describir los factores de riesgo encontrados con más frecuencia en pacientes con vejiga neurógena secundaria a lesión de la médula espinal.

- Caracterizar el comportamiento microbiológico de los uropatógenos aislados en estos pacientes.

- Describir el patrón de sensibilidad/resistencia de las principales bacterias aisladas.

- Describir las cepas bacterias resistentes en nuestra clínica.

\section{MATERIAL Y MÉTODOS}

Realizamos un estudio descriptivo retrospectivo en todos los pacientes hospitalizados en la clínica de lesionados medulares para realizar un tratamiento neuroregenerativo de la enfermedad en un período de seis meses. Veintiocho pacientes fueron incluidos; de ellos el 57,1\% eran adultos jóvenes entre 15-30 años, un 32,1\% tenían entre 31-45 y sólo el 10,7\% estaban por encima de esta edad. Dieciocho pacientes eran parapléjicos y 10 cuadripléjicos. Quince eran varones y 13 mujeres.

Todos los pacientes fueron sometidos a evaluación clínica, técnicas de imagen del aparato urinario (ultrasonidos y cistouretrografia) y estudios microbiológicos: cultivos de orina, exudado uretral y vaginal utilizando la prueba Diramic ${ }^{15-16}$. Es necesario mencionar que todas las muestras para cultivo se tomaron con catéteres estériles cambiados o colocados en las seis horas previas, en el caso de pacientes con sonda permanente, o en el caso de cateterismo intermitente, reduciendo así el número de aislamientos y contaminación de las muestras ${ }^{17}$.

Determinamos los factores de riesgo, las formas de presentación de la infección, así como las complicaciones asociadas y el comportamiento microbiológico de los gérmenes en veintiocho pacientes con criterios clínicos y microbiológicos de infección del tracto urinario intrahospitalaria $^{7,18}$; se cuantificaron también las cepas de uropatógenos multiresistentes aisladas.

\section{RESULTADOS Y CONCLUSIONES}

En nuestros pacientes, las manifestaciones clínicas típicas de infección del tracto urinario sintomática están generalmente ausentes debido 
al compromiso de su sensibilidad, y los signos y síntomas sugestivos de infección urinaria en pacientes con lesión medular espinal incluyen fiebre, malestar o dolor en la zona lumbar o hipogástrica, incontinencia urinaria, aumento de la espasticidad, hiperreflexia autonómica, orina turbia con olor, malestar, letargo o sensación de mareo, similares a otros estudios ${ }^{10,12}$.

La forma más frecuente de presentación de infección urinaria (Fig. 1) fue la infección urinaria sintomática, específicamente bacteriuria sintomática que apareció en el $67 \%$ de los pacientes e infección del tracto urinario superior, pielonefritis, presente en el $17,8 \%$ de los pacientes, paradójicamente predominante en varones a diferencia de lo que sucede en la población normal un asunto discutible porque en la muestra predominan los varones-; además, el 50\% de las pacientes lesionadas medulares $(6 / 12)$ presentaban vaginosis bacteriana, que puede estar etiopatogénicamente relacionada con los episodios de bacteriuria sintomática que presentan $(9 / 12)$. Estos hechos se explican perfectamente por el riesgo adicional del aparato urinario de la mujer, "riesgo anatomofisiológico" con una uretra más corta, relaciones sexuales e incontinencia rectal, que en el caso de la mujer tienen una repercusión mayor en el sistema urogenital. El agente etiológico predominante en tales episodios es E.coli, hallado en el 60\% de los casos (Fig. 2) y otras

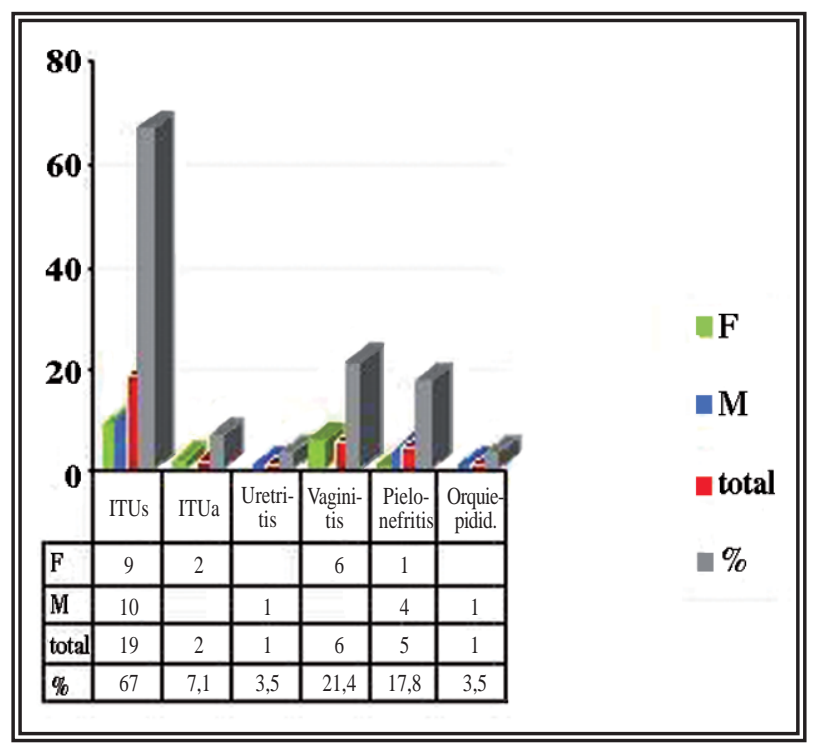

FIGURA 1. Presentación clínica de la infección urinaria en pacientes lesionados medulares espinales.

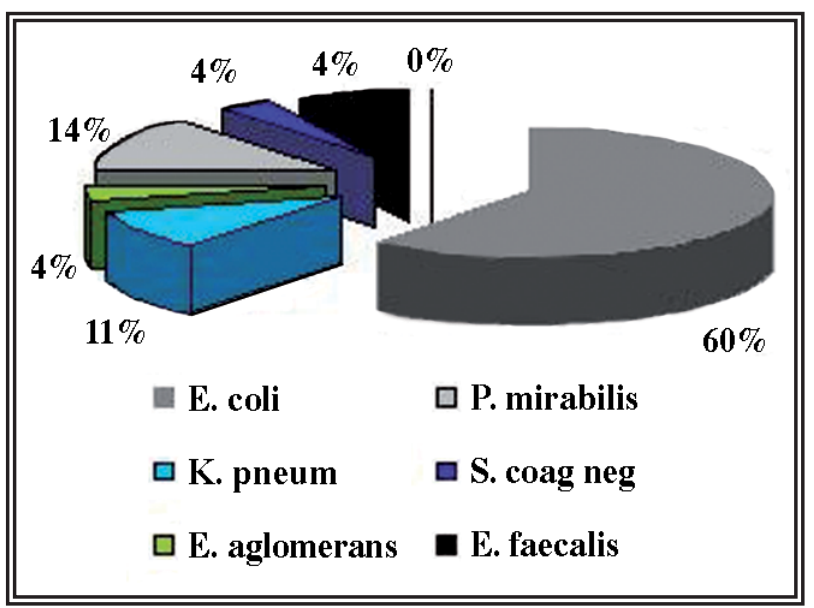

FIGURA 2. Uropatógenos aislados en pacientes con vejiga neurógena einfección urinaria intrahospitalaria.

enterobacterias, como K. pneumoniae y P. mirabilis principalmente; cuando se compara con ITU en pacientes sin lesión medular en otras publicaciones no hay diferencias remarcables en uropatógenos $^{19-20}$. La orquioepididimitis y la uretritis eran menos frecuentes en este estudio $(1 / 28)$, porque en este grupo de edad se relacionan frecuentemente con otras etiologías como enfermedades de transmisión sexual, siendo los principales agentes causantes chlamidia tracomatis y neisseria gonorrhoeae $e^{21}$.

Los riesgos más frecuentes relacionados con infección urinaria fueron en orden descendente (Tabla 1): utilización de cateterismo intermitente, realizado por el $75 \%$ de los pacientes, realización previa de algún procedimiento diagnóstico como cistografia miccional $(21,4 \%)$, así como la utilización de sonda permanente, la presencia de diver-

Tabla 1. Factor de riesgo en lesionados medulares espinales con infección urinaria.

\begin{tabular}{lcc}
\hline $\begin{array}{l}\text { Factor } \\
\text { de riesgo }\end{array}$ & $\begin{array}{c}\text { No de } \\
\text { pacientes }\end{array}$ & \% \\
\hline Catéter permanente & 2 & 7,14 \\
Divertícula y pseudodivertícula & 2 & 7,14 \\
Piedras vesicales o renales & 2 & 7,14 \\
Cistografia urinaria previa & 6 & 21,4 \\
Cateterización intermitente & 21 & $75 \%$ \\
Procediemiento quirúrgico & 1 & 3,5 \\
Inmunodepresión & 1 & 3,5 \\
Solamente vejiga neurogénica & 3 & 10,5 \\
\hline
\end{tabular}


tículos en la pared vesical y la litiasis vesical (respectivamente $7,1 \%$ ) o cualquier otro factor como cirugía previa, inmunodepresión y vejiga neurógena con cualquier otra complicación aparente, aunque existen otros factores que influyen y pueden predecir el deterioro del tracto urinario superior en pacientes con vejiga hiperactiva tales como presión de fuga del detrusor, duración de la enfermedad, tiempo de cateterización, grado de inflamación y severidad de la fibrosis vesical ${ }^{22}$, que no han sido estudiados en este grupo.

Con respecto a la susceptibilidad de los uropatógenos (Fig. 3), los E. coli hallados mantienen una buena sensibilidad a amikacina y nitrofurantoina (> 90\%), aunque hay resistencias crecientes a gentamicina (25\%), que ha sido el aminoglucósido de elección para enterobacterias. Sin embargo, se han comunicado resistencias a sulfamidas mediadas por plásmidos en $E$. coli en el $70 \%$, lo cual se considera en general bastante alto en comparación con otras comunicaciones sobre resistencias a cotrimoxazol en uropatógenos ${ }^{23}$. Por ejemplo, en algunos estados de los Estados Unidos han cambiado desde el 33\% al 43\%; en España e Israel aproximadamente al $30 \%$ y al $60 \%$ en Bangladesh, aunque debemos decir que las muestras positivas de nuestro estudio provenían sólo de episodios de infección intrahospitalaria en pacientes con infecciones complicadas. Se trata de pacientes con vejiga neurógena, con el antecedente de uso frecuente de antimicrobianos, y se acepta así una razón

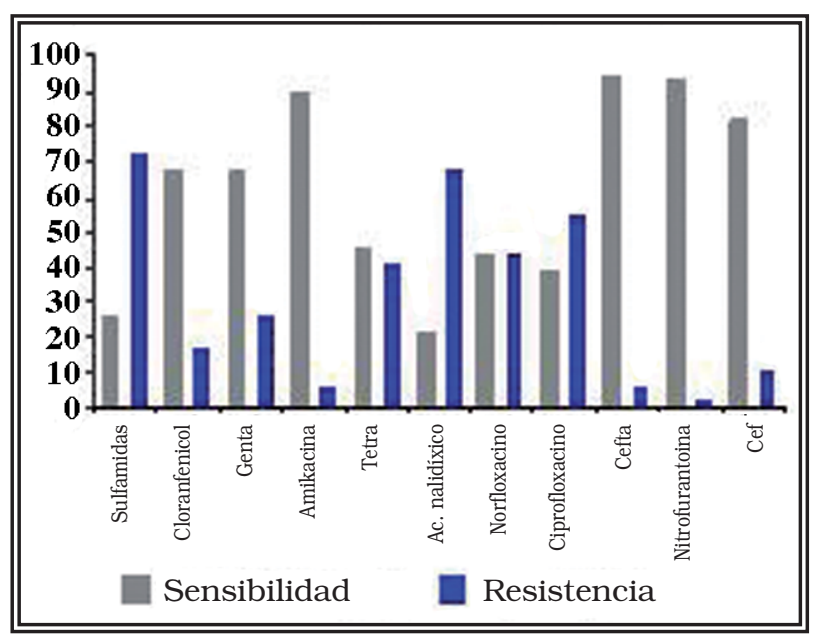

FIGURA 3. Patrón de Sensibilidad/Resistencia en cultivos de orina (E. coli). para presentar una mayor resistencia en comparación con otras publicaciones ${ }^{24-26}$ con muestras de orina proveniente de infecciones no complicadas.

También se observó una importante y progresiva resistencia a quinolonas que varía entre el $67 \%$ del ácido nalidíxico, 55\% de ciprofloxacino y $43 \%$ de norfloxacino, que también es alta en comparación con otras publicaciones ${ }^{27-28}$. Es un asunto de importancia epidemiológica institucional, si consideramos que es una familia de antimicrobianos de elección en estos episodios infecciosos. El mecanismo de resistencia implicado más frecuentemente se ha relacionado con mutaciones que pueden ocurrir en la subunidades de DNA girasa, descritas en algunas enterobacterias como E. coli, o en porinas, proteínas de las membranas celulares que reducen la entrada del fármaco en la bacteria, lo cual disminuye naturalmente su eficacia y aumenta la resistencia al tratamiento. Este es un asunto para estudios futuros. Estos son algunos resultados después de llevar a cabo un programa por la comisión de control y prevención de infecciones institucionales en relación con la política de utilización de antimicrobianos con restricción o ciclos repetidos de algunas de estas familias en infección urinaria, así como la prohibición de su utilización empírica y en profilaxis antibiótica ${ }^{29-31}$.

La circulación de patógenos resistentes a varias familias de antimicrobianos, sobre todo quinolonas, sulfamidas, betalactámicos y tetraciclinas, es un fenómeno también descrito en nuestro estudio, donde predominan los E. coli. Un fenómeno ya descrito hace más de una década en infecciones urinarias complicadas, que se presentó hasta en el $20 \%$ de los gérmenes aislados y que por lo tanto causó episodios de recurrencia de bacteriuria y frecuentes fallos terapéuticos, incluso cuando la sensibilidad a quinolonas era alta $^{27}$. Este hecho podría estar relacionado con la frecuente combinación de mecanismos de resistencia mostrada por las bacterias gram negativas, que siendo resistentes a quinolonas por medio de RNA girasa y mutaciones de porinas, desarrollan resistencias cruzadas a otras familias de antimicrobianos. A pesar de saber que las fluorquinolonas se utilizan con más frecuencia en infecciones urinarias, como estos mecanismos 
de entrada al microorganismo también los utilizan las tetraciclinas y el cloranfenicol; ésta podría ser una explicación plausible ${ }^{32-33}$.

Sin embargo, siendo la vigilancia y comunicación un objetivo permanente dentro de sistema de vigilancia epidemiológica se observa una tendencia creciente de la circulación de patógenos multirresistentes en nuestro ambiente, principalmente uropatógenos (Fig. 4): 9,4\% en 2001, 5,9\% en 2002, $7,2 \%$ en $2003,22 \%$ en 2004 y $13 \%$ en 2005 ; esto contribuye al dificil manejo de los episodios de infección en estos pacientes y a las infecciones complicadas, persistentes y recurrentes que hacen que aumente el coste, la morbilidad y también dificultan el rendimiento físico de los pacientes durante el tratamiento intensivo de rehabilitación que realizan ${ }^{34}$. En el 2004 fue necesario que la comisión hospitalaria de control de antibióticos llevara a cabo una intervención en relación, con proscripción del uso de sulfas y quinolonas en infección urinaria nosocomial durante un año, y en el año 2005 vimos una disminución que la circulación de cepas de uropatógenos resistentes.

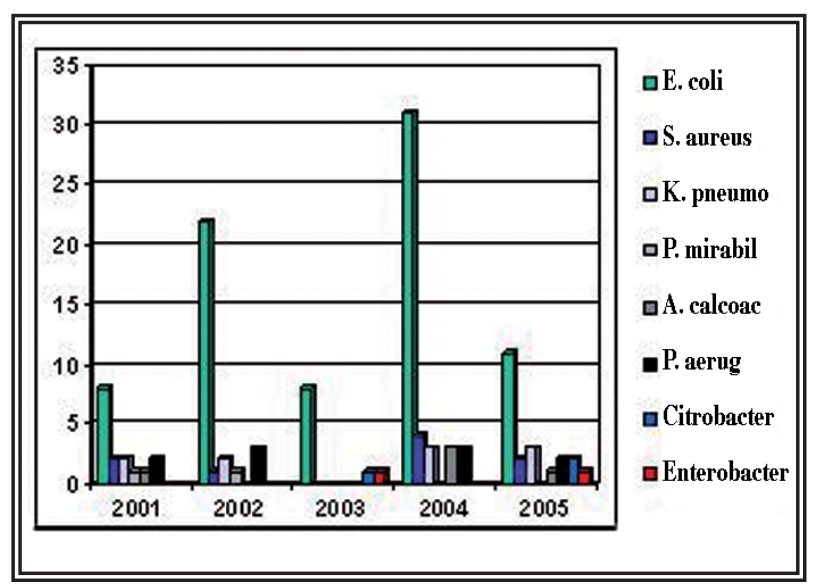

FIGURA 4. Cepas bacterianas multirresistentes.

La aparición de uropatógenos resistentes en el ambiente hospitalario es por sí misma un fenómeno epidemiológico destacado que ha traído nuevas estrategias para su manejo ${ }^{35-36}$ también utilizadas en nuestro ambiente como medida de control de infecciones.

\section{REFERENCIAS}

1. Prevalencia de infecciones nosocomiales en hospitales españoles, Estudio EPINE. [Sitio en Internet] Disponible en: Con acceso el 26 de abril de 2005.
12. Harrop JS, Sharan AD, Przybylski GJ. Epidemiology of Spinal Cord Injury After Acute Odontoid Fractures. Neurosurg Focus 8(6).

13. Nickel JC, Costerton JW, McLean RJ. Bacterial biofilms: influence on the pathogenesis, diagnosis and treatment of urinary tract infections. J Antimicrob Chemother. 1994;33 (suppl A):31-41.

14. Kennedy RH. The new viewpoint toward spinal cord injuries. Ann Surg. 1946;124: 1057-1065

15. Kass EH. Chemotherapeutic and antibiotic drugs in the management of infections of the urinary tract. Am J Med. 1955; 18(5):764-781.

16. Surkin J, Smith M, Penman A, Currier M, Harkey HL III, Chang YF Spinal Cord Injury Incidence in Mississippi: A Capture-Recapture Approach. J Trauma. 1998 Sep;45(3): 502-504.

17. Gribble MJ, McCallum NM, Schechter MT. Evaluation of diagnostic criteria for bacteriuria in acutely spinal cord injured patients undergoing intermittent catheterization. Diagn Microbiol Infect Dis 1988; 9(4):197-206.

18. Stark RP, Maki DG. Bacteriuria in the catheterized patient: what quantitative level of bacteriuria is relevant? N Engl J Med. 1984; 311(9):560-564.

19. National Institute on Disability and Rehabilitation. The prevention and management of urinary tract infections among people with spinal cord injuries: National Institute on Disability and Rehabilitation Research Consensus Statement. J Am Paraplejia Soc 1992; 15: 194-204.

10. Esclarín De Ruz A, García Leoni E, Herruzo Cabrera R.. Epidemiology and Risk factor for urinary tractus infeccion in patients with spinal cord injure. J Urol. 2000;164(4): 1285-1289.

11. Foxman B. Epidemiology of urinary tract infections: incidence, morbidity, and economic costs. Am J Med. 2002 Jul 8;113 Suppl 1A:5S-13S.

12. Penders J, Huylenbroeck AA, Everaert K, Van Laere M, Verschraegen GL. Urinary infections in patients with spinal cord injury. Spinal Cord. 2003 Oct;41(10):549-552.

13. Kaufman JM, Fam B, Jacobs SC, Gabilondo F, Yalla S, Kane JP, Rossier AB: Bladder cancer and squamous metaplasia in spinal cord injury patients. J Urol. 1977;118(6): 967-997.

14. Subramanian Vaidyanathan, Paul Mansour, Bakul M Soni, Gurpreet Singh and Pradipkumar Sett. The method of bladder drainage in spinal cord injury patients may influence the histological changes in the mucosa of neuropathic bladder -a hypothesis. BMC Urology 2002;2:5.

15. Contreras OR, Roura G, Fernandez D Diagnosis from urine culture in 4 hours using the DIRAMIC-03 system.Rev Latinoam Microbiol. 1992; 34(2):83-86.

16. Alarcón RC, Ruiz FT, Tamayo AZ, Carmona GR, Varela EA, Ochoa GT, Frómeta NR. Mycrob-1000: an alternative for the rapid determination of urine culture in the primary health level]. Rev Cubana Med Trop, Mayo-ago. 2004;56 (2):135-138.

17. Shah PS, Cannon JP, Sullivan CL, Nemchausky B, Pachucki CT. Controlling Antimicrobial Use and Decreasing Microbiological Laboratory Tests for Urinary Tract Infections in Spinal-CordInjury Patients with Chronic Indwelling Catheters. Am J Health-Syst Pharm. 2005; 62(1):74-77.

18. WHO/CDS/CSR/EPH/2002. Prevención de las infecciones nosocomiales. Guia Práctica. [Sitio en Internet] Disponible en: http://www.who.int/csr/resources/publications/drugresist/en/PISpanish3.pdf. Con acceso el 14 de julio de 2005. 
19. Bennett CJ, Young MN, Darrington H. Differences in urinary tract infections in male and female spinal cord injury patients on intermittent catheterisation. Paraplegia. 199; 33(2):69-72.

20. Waites KB, Canupp KC, DeVivo MJ. Microbiology of the urethra and perineum and its relationship to bacteriuria in community-residing men with spinal cord injury. 2004; 27(5):448-52.

21. Delavierre D. Orchi-epididymitis. Ann Urol (Paris). 2003; 37(6):322-338.

22. Ozkan B, Demirkesen O, Durak H, Uygun N, Ismailoglu V, Cetinel B. Which factors predict upper urinary tract deterioration in overactive e neurogenic bladder dysfunction? Urology. 2005 Jul;66(1):99-104.

23. Ken Waites. Epidemiology of Antimicrobial Resistance in Spinal Cord Injury. Volume 16, Number 2: Summer, 1998. 619 19th St S, Birmingham, AL 35249-7330.

24. Sahm DF, Thornsberry C, Mayfield DC, Jones ME, Karlowsky JA. Multidrug-resistant urinary tract isolates of Escherichia coli: prevalence and patient demographics in the United States in 2000. Antimicrob Agents Chemother. 2001;45(5):1402-1406.

25. Jacoby G, Archer G. New Mechanisms of Bacterial Resistance to Antimicrobial Agents. New Engl J Med. 1991;324: 601 .

26. Muder RR, Brennen C, Drenning SD, Stout JE, Wagener MM. Multiple antibiotic-resistant gram-negative bacilli in a long-term-care facility: a case-control study of patient risk factors and prior antibiotic use. Infect Control Hosp Epidemiol. 1997;18(12):809-813.

27. Yoshida H., Bogaki M., Nakamura M., \& Nakamura S. Quinolone resistance-determining region in the DNA gyrase gyrA gene of Escherichia coli. Antimicrob. Agents Chemother. 1990;34(6):1271-1972.

28. Schaeffer AJ. Review of norfloxacin in complicated and recurrent urinary tract infections. Eur Urol. 1990;17 Suppl. 1: 19-23.

29. Estrategia mundial OMS de contención de la resistencia a los antimicrobianos - Resumen. WHO/CDS/CSR/DRS/ $2001.2 \mathrm{a}$
30. Erik R. Dubberke, MD, Victoria J. Fraser, MD. Cycling and Other Strategies to Slow and Reverse Antibiotic Resistance, Washington University School of Medicine, St Louis Infect Med. 2004;21(11):544-556.

31. Stelling J, Travers K, Jones R, Philip PJ, O’Brien T, and Levy SB. Integrating Escherichia coli Antimicrobial Susceptibility Data from Multiple Surveillance Programs. Emerging Infectious Diseases. Current Issue. 2005. 11(6): 873-882.

32. Goettsch W, van Pelt W, Nagelkerke N, Hendrix MG, Buiting $\mathrm{AG}$, Petit PL. Increasing resistance to fluoroquinolones in Escherichia coli from urinary tract infections in the Netherlands. J Antimicrob Chemother. 2000;46(2): 223-228.

33. Yamane K, Wachino J, Doi Y, Kurokawa H and Arakawa Y. Global Spread of Multiple Aminoglycoside Resistance Genes. Vol. 11, No. 6 June 2005 Emerging Infectious Diseases Vol. 11, No. 6, June 2005. [Sitio en internet]. con acceso 9 de marzo 2006].

34. Shlaes DM, Gerding DN, John JF, Craig WA, Bornstein DL, Duncan RA, et al.Society for Healthcare Epidemiology of America and Infectious Diseases Society of America Joint Committee on the Prevention of Antimicrobial Resistance: guidelines for the prevention of antimicrobial resistance in hospitals. Clin Infect Dis. 1997;25:584-599.

35. Fridkin SK. Routine cycling of antimicrobial agents as an infection-control measure. Clin Infect Dis. 2003;36:14381444 .

36. Lindsay E.Thomas M. Wanda K. Fisher C;Fourcroy C, Gupta K, et al. Managing Acute Uncomplicated Cystitis in the Era of Antibiotic Resistance. [Sitio en Internet] Medscape. Disponible en: http://www.medscape.com/ iewprogram/2634_pnt. con acceso 10 de mayo 2005.

Correspondencia autora: Dr. E. Hernández Fernández Specialist of Spinal Cord Injure Clinic, Infectologist Ave 25 No15805, e/ 158 y 160. Cubanacan, Playa, CH Cuba CP 11300. Tel.: 53-7 273 6777, 6778

E-mail autora: elizabeth.hdez@infomed.sld.cu Información artículo: Original - Vejiga neurógena Trabajo recibido: septiembre 2006

Trabajo aceptado: mayo 2007 